\title{
Perioperative Management of an Adolescent With Friedreich's Ataxia and Hypertrophic Cardiomyopathy
}

\author{
Mineto Kamata ${ }^{\mathrm{a}}$, David Martin ${ }^{\mathrm{a}, \mathrm{b}}$, Allan Beebe ${ }^{\mathrm{c}}$, Walter Samora ${ }^{\mathrm{c}}$, \\ Aymen Naguib ${ }^{\mathrm{a}, \mathrm{b}}$, Joseph D. Tobias ${ }^{\mathrm{a}, \mathrm{b}, \mathrm{d}, \mathrm{e}}$
}

\begin{abstract}
Friedreich's ataxia (FA) is an autosomal recessive neurodegenerative disorder with an incidence of 1:50,000 which usually presents during adolescents. Given the progressive neurologic involvement, surgical intervention is frequently required for correction of orthopedic sequelae including scoliosis. Associated co-morbid conditions including hypertrophic cardiomyopathy, restrictive lung disease, and diabetes mellitus may impact the perioperative care of such patients. We present a 14-year-old girl with FA presenting for posterior spinal fusion for the treatment of scoliosis. The perioperative management of such patients is discussed.
\end{abstract}

Keywords: Friedreich's ataxia; Hypertrophic cardiomyopathy; Posterior spinal fusion; Scoliosis

\section{Introduction}

Friedreich's ataxia (FA) is an uncommon $(1: 50,000)$, autosomal recessive neurodegenerative disorder which usually presents during adolescents. Initial symptoms include an unsteady posture, frequent falling, and progressive difficulties with ambulation due to an impaired ability to coordinate voluntary movements. Pathologic findings include progressive

\footnotetext{
Manuscript accepted for publication July 30, 2014

${ }^{a}$ Department of Anesthesiology \& Pain Medicine, Nationwide Children's Hospital, Columbus, OH, USA

${ }^{b}$ Department of Anesthesiology \& Pain Medicine, The Ohio State University College of Medicine, Columbus, OH, USA

${ }^{\mathrm{c}}$ Department of Orthopedic Surgery, Nationwide Children's Hospital \& The Ohio State University College of Medicine, Columbus, OH, USA

${ }^{\mathrm{d}}$ Department of Pediatrics, Nationwide Children's Hospital \& The Ohio

State University College of Medicine, Columbus, OH, USA

${ }^{\mathrm{e}}$ Corresponding Author: Joseph D. Tobias, Department of

Anesthesiology \& Pain Medicine, Nationwide Children's Hospital, 700

Children's Drive, Columbus, OH 43205, USA.

Email: Joseph.Tobias@Nationwidechildrens.org

doi: http://dx.doi.org/10.14740/jmc1900w
}

degeneration and atrophy of neurons in the spinocerebellar and pyramidal tracts, atrophy of dorsal root ganglia, and cerebellar dysfunction [1]. Cognitive function is maintained. Although the diagnosis previously rested on clinical and radiographic findings, genetic testing is now feasible for the defective gene $[1,2]$. Associated co-morbid conditions may include hypertrophic cardiomyopathy (HCM) and diabetes mellitus.

The primary pathophysiologic process resulting in FA is mutation of the gene coding for the mitochondrial protein, frataxin [2]. Although the exact role of frataxin in normal physiology remains unclear, the frataxin decrease results in mitochondrial iron overload leading to disruption of mitochondrial function and cell death. Given the progressive neurologic involvement, surgical intervention is frequently required for correction of orthopedic sequelae. We present a 14-year-old girl with FA presenting for posterior spinal fusion (PSF) for the treatment of scoliosis. The perioperative management of such patients with emphasis on the care of patients with HCM is discussed.

\section{Case Report}

A 14-year-old girl weighing $37.2 \mathrm{~kg}$ presented for general anesthesia for PSF. Her past history was significant for frequent falls and an abnormal gait starting at $8-9$ years of age. These symptoms had worsened gradually over the ensuing years and led to genetic testing and identification of her condition as FA at 14 years of age. Echocardiogram 2 months prior to surgery showed concentric moderate to severe left ventricular hypertrophy, mild mitral insufficiency, and normal right and left ventricular systolic function. Cardiac MRI 2 weeks prior to surgery revealed moderate asymmetric septal hypertrophy with mildly elevated left ventricular mass, normal bi-ventricular systolic function, and mild mitral valve insufficiency without evidence of myocardial delayed enhancement. Her medication regimen included montelucast 5 $\mathrm{mg}$ and cetirizine $10 \mathrm{mg}$ once a day. Preoperative laboratory evaluation showed a hemoglobin of $14.0 \mathrm{~g} / \mathrm{dL}$, a hematocrit of $40.8 \%$, and a platelet count of $280,000 / \mathrm{mm}^{3}$. Electrolytes and serum glucose were within normal limits. The patient 
arrived to the preoperative area from home on the morning of surgery with a room air oxygen saturation of $100 \%$. A 20 gauge intravenous cannula was placed in the surgical unit. Following premedication with intravenous midazolam $(0.05$ $\mathrm{mg} / \mathrm{kg}$ ), she was transported to the operating room. Anesthesia was induced with etomidate $(0.25 \mathrm{mg} / \mathrm{kg})$, lidocaine (1 $\mathrm{mg} / \mathrm{kg})$, and fentanyl $(3 \mu \mathrm{g} / \mathrm{kg})$. Endotracheal intubation was facilitated by rocuronium $(0.5 \mathrm{mg} / \mathrm{kg})$. Prior to and during anesthetic induction, esmolol was administered in $0.25 \mathrm{mg} /$ $\mathrm{kg}$ increments (total of 3 ) to lower the heart rate (HR) from 110 beats $/ \mathrm{min}$ to $60-70$ beats/min. There was no change in the blood pressure (BP) during anesthetic induction. Direct laryngoscopy was performed and when there was no change in the hemodynamic parameters, a $6.0 \mathrm{~mm}$ cuffed endotracheal tube was placed. A second 16 gauge intravenous cannula and a 20 gauge radial arterial cannula were placed. Using ultrasound guidance, a 4 French, $12 \mathrm{~cm}$ double lumen catheter was placed in the right internal jugular vein. Additional monitoring included near infrared spectroscopy (NIRS) to monitor cerebral oxygenation and systemic oxygenation (deltoid muscle) and the bispectral index (BIS). Initial NIRS values varied from $65 \%$ to $80 \%$ in both sites. Anesthesia was maintained with sevoflurane (end-tidal concentration 2-3\%) to maintain the BIS at 50 - 60 and a remifentanil infusion $(0.05-0.3 \mu \mathrm{g} / \mathrm{kg} / \mathrm{min})$. A phenylephrine infusion $(0.2-0.5 \mu \mathrm{g} / \mathrm{kg} / \mathrm{min})$ was titrated to maintain the mean arterial pressure (MAP) at $55-65 \mathrm{~mm} \mathrm{Hg}$. Following anesthetic induction and placement of invasive arterial and central venous cannulae, a dexmedetomidine infusion was started at $0.4 \mu \mathrm{g} / \mathrm{kg} / \mathrm{h}$ without a loading dose. Neurophysiological monitors were placed for the intraoperative monitoring of motor and somatosensory evoked potentials. The patient was positioned prone and a forced air warming blanket was placed. Tranexamic acid was administered (bolus dose of $50 \mathrm{mg} / \mathrm{kg}$ followed by an infusion of $5 \mathrm{mg} / \mathrm{kg} / \mathrm{h}$ during the case). Prior to the start of the surgical procedure, $250 \mathrm{~mL}$ of $5 \%$ albumin was administered to replace the fluid deficit and increase intravascular volume. Initial arterial blood gas analysis was unremarkable with a venous saturation (blood obtained from the central venous catheter) of $72 \%$ and a lactate concentration of $0.93 \mathrm{mmol} / \mathrm{L}$. During the operation, the MAP was maintained at 55 - $65 \mathrm{~mm} \mathrm{Hg}$. PSF with instrumentation was performed from the $\mathrm{T}_{2}$ to the $\mathrm{L}_{3}$ level. During closure of the surgical incision, the hemoglobin was $8.2 \mathrm{~g} / \mathrm{dL}$ and the venous saturation was $84 \%$. Intraoperative salvaged blood (volume of $225 \mathrm{~mL}$ ) was subsequently returned to the patient. Acetaminophen $(15 \mathrm{mg} / \mathrm{kg})$ and hydromorphone $(0.03 \mathrm{mg} / \mathrm{kg}$ increments) were administered for postoperative analgesia. The remifentanil and phenylephrine infusions and the inhaled sevoflurane were discontinued. The patient was turned to the supine position. An esmolol infusion (75 $\mu \mathrm{g} / \mathrm{kg} / \mathrm{min}$ ) was started at the end of the case to maintain the HR at 60 - 80 beats $/ \mathrm{min}$. The dexmedetomidine infusion was continued at $0.4 \mu \mathrm{g} / \mathrm{kg} / \mathrm{h}$. The patient's trachea was ex- tubated in the operating room and she was transported to the cardiothoracic intensive care unit. The surgical duration was $360 \mathrm{~min}$. Total blood loss was estimated at $500 \mathrm{~mL}$ and urine output was 1,190 mL. Total fluids included 2,100 mL of isotonic crystalloid, $1,000 \mathrm{~mL}$ of $5 \%$ albumin, and $225 \mathrm{~mL}$ of intraoperative cell salvage. Postoperative analgesia was provided by patient controlled analgesia with hydromorphone. Intravenous acetaminophen was administered every $6 \mathrm{~h}$ for the initial 48 postoperative hours. The dexmedetomidine and esmolol infusions were continued until the next morning when the central venous catheter was removed. Hemoglobin and hematocrit reached a postoperative nadir of $8.7 \mathrm{~g} / \mathrm{dL}$ and $25.9 \%$ respectively on postoperative day 1 without the administration of allogeneic blood products. The remainder of her postoperative course was uncomplicated.

\section{Discussion}

Given its multi-system involvement, there may be several co-morbid conditions which impact the perioperative care of patients with FA. The presentation of FA may vary significantly including involvement primarily of fine motor movements and gait with unsteady posture, frequent falling, and progressive difficulties with ambulation [3]. PSF for the treatment of progressive scoliosis is necessary in up to onethird of patients with FA [4].

Given the high incidence of cardiac involvement even without clinical symptoms, preoperative electrocardiography and echocardiography is suggested in all patients with FA. Cardiomyopathy remains the primary cause of death in FA [2]. Cardiac involvement resulting in the need for cardiac transplantation has been reported prior to the onset of neurological symptoms of FA [5]. HCM can be detected by echocardiography in approximately $65 \%$ of patient, but more subtle abnormalities can be detected in almost all FA patients [6-8]. Arrhythmias are common and often intractable in HCM. They may be of atrial or ventricular origin, impacting ventricular preload and leading to hemodynamic compromise. If there are any clinical concerns regarding arrhythmias or findings on the preoperative ECG, more thorough investigations including 24-h Holter monitoring or electrophysiological studies may be indicated prior to anesthetic care. Given the complex nature of our patient's co-morbid cardiac disease, close collaboration with our pediatric cardiology colleagues was required. This included not only noninvasive imaging, but also preoperative discussion regarding the optimal intraoperative management.

The major goals of perioperative care are to avoid tachycardia, maintain normal sinus rhythm, avoid increases in myocardial contractility, and maintain preload and afterload. In our patient, we chose to place an intravenous cannula prior to anesthetic induction to allow for close control of these parameters during anesthetic induction. Fluctuations 
are particularly common during anesthetic induction, direct laryngoscopy, and endotracheal intubation. Hemodynamic stability was facilitated by anesthetic induction with etomidate, fentanyl and lidocaine [9]. Although its adrenal suppressive effects due to inhibition of 11- $\beta$ hydroxylase have made its use controversial in specific clinical scenarios, adverse perioperative implications of this effect have not been clearly demonstrated. Additional control of the perioperative hemodynamic parameters (maintenance of a low HR and a high-normal systemic vascular resistance) was facilitated by the intraoperative use of esmolol and phenylephrine. An additional concern perioperatively with HCM is the potential for an imbalance of the myocardial oxygen delivery-demand ratio. Histologically, the thickened walls of $\mathrm{MCH}$ may result in luminal narrowing of the intramural coronary arteries.

Maintenance anesthesia included a technique that incorporated the synthetic opioid, remifentanil, sevoflurane, and dexmedetomidine. These agents provided adequate intraoperative anesthesia, blunted the sympathetic stress response, allowed for early tracheal extubation, and provided appropriate conditions for intraoperative monitoring of somatosensory/motor evoked potentials. Sevoflurane was chosen instead of desflurane to avoid tachycardia and stimulation of the sympathetic nervous system which may be seen with desflurane.

Given the associated co-morbid cardiac disease and the magnitude of the surgical procedure, intraoperative monitoring included invasive monitoring of both arterial blood pressure and central venous pressure. In the surgical setting with a patient placed in the prone position, CVP monitoring may be inaccurate $[10,11]$. Intraoperative transesophageal echocardiography (TEE) may be warranted in this setting to ensure proper volume management and hemodynamic stability. Given patient positioning (prone position with slight neck flexion), we have concerns regarding lingual edema related to the prolonged placement of a TEE probe. In addition to TEE and CVP monitoring, we suggest mixed-venous oxygen saturation $\left(\mathrm{SvO}_{2}\right)$ and monitoring of plasma lactate concentrations to evaluate end-organ oxygen delivery [12]. We have also found utility in the use of NIRS to monitor cerebral oxygenation as an indicator of end organ perfusion [13]. NIRS probes can be placed over the forehead to monitor cerebral oxygenation and over a peripheral muscular bed (deltoid in our patient) to measure peripheral tissue oxygenation.

The involvement of the central nervous system may result in implications regarding the use of neuromuscular blocking agents during anesthetic care in patients with FA. Previous reports caution against the use of succinylcholine in patients with FA given there is progressive limb and gait ataxia with skeletal muscle weakness and the theoretical risk of a hyperkalemic response following succinylcholine [14]. The involvement of the central and peripheral nervous system would also theoretically make these patients sensitive to the effects of non-depolarizing NMBAs. Alternatively, en- dotracheal intubation can also be accomplished without the use of a NMBA with agents such as propofol in combination with either sufentanil or remifentanil $[15,16]$. In our patient, we used rocuronium to facilitate endotracheal intubation. Although we did not believe that it would be clinically significant, given that we were controlling HR during induction with esmolol, it should be recognized that there may be a mild vaoglytic response with rocuronium with a resultant increase in HR which may be detrimental in the setting of HCM.

Approximately $10 \%$ of FA patients are diabetic and may require insulin therapy during the perioperative period. Preoperative evaluation is suggested with routine screening of fasting blood glucose during the preoperative visit as well as questioning regarding clinical signs and symptoms such as polyuria and polydypsia. Even in the absence of clinical findings, there remains the potential for the perioperative stress response to precipitate diabetic ketoacidosis so careful postoperative monitoring is required. In patients with glucose intolerance, perioperative control may be indicated as hyperglycemia may impair wound healing and increase the risk of surgical site infections.

While clinical studies have not consistently demonstrated a significant relationship between perioperative glycemic control and short-term risk of infection or morbidity, tight glucose control has been recommended by others. Additionally, hyperglycemia may result in glucosuria, polyuria, and electrolyte disturbances. Given these concerns, appropriate control of intraoperative glucose homeostasis may be beneficial. For major surgical procedures, a continuous intravenous infusion of insulin may be superior to subcutaneous injections in achieving perioperative optimal glycemic control. Given the complexity and variability of current diabetes treatment options, consultation with the pediatric endocrinology service should be considered.

PSF may result in significant intraoperative blood loss, at times in excess of an entire blood volume. The prone position increases intra-abdominal pressure, which leads to compression of the inferior vena cava. The compressed vena cava impedes venous return to the heart, thereby decreasing cardiac output and engorging epidural veins. Effective positioning on the Jackson table may help limit abdominal and vena caval compression thereby decompressing epidural veins. Multiple other strategies to minimize intraoperative blood loss and allogeneic transfusions can be employed during major orthopedic procedures including autologous blood donation, normovolemic hemodilution, intraoperative blood salvage, controlled hypotension, and manipulation of the coagulation cascade with DDAVP, anti-fibrinolytic agents (epsilon-aminocaproic acid, tranexamic acid, and aprotinin) or recombinant factor VIIa [17]. In our clinical practice, the commonly used techniques include controlled hypotension, tranexamic acid as an anti-fibrinolytic agent, and intraoperative cell salvage. As the accurate assessment of blood loss 
may be impossible, the decision to transfuse blood products should be based on the balance between oxygen delivery and demand using the combination of several factors including hemoglobin concentration, hemodynamic status, peripheral and central oxygen saturation (NIRS), and acid-base status including lactic acid determinations.

Another concern in patients with neurological disabilities is the potential for postoperative respiratory failure or insufficiency. As the degree of curvature progresses, scoliosis impedes on the movement of the ribs, and places the respiratory muscles at a mechanical disadvantage with a decrease in the compliance of the chest wall directly and the lung indirectly. Scoliosis surgery produces an immediate and transient decrease in vital capacity of up to $40 \%$ in virtually all patients even those with idiopathic scoliosis without co-morbid conditions. These factors may result in postoperative respiratory insufficiency or failure with the need for prolonged postoperative mechanical ventilation. Patients who have abnormal results on their pulmonary function test, particularly a forced vital capacity of less than $30 \%$, or those who have hypercapnia preoperatively have been shown to have a higher need for postoperative ventilation [18]. Given these concerns, preoperative optimization of pulmonary function is suggested. Preoperative preparation should include the treatment of respiratory infections and instructions regarding the use of incentive spirometry with the consideration of extubation to non-invasive ventilation techniques [19].

During the postoperative period, the management focuses on providing adequate pain control while minimizing the respiratory depressant effects of opioids. Postoperative analgesia for our patient was achieved by patient-controlled analgesia with hydromorphone. Adjunctive agents including acetaminophen or non-steroidal anti-inflammatory agents should be incorporated into the postoperative analgesic regimen. Regardless of the agents used, postoperative monitoring of respiratory function is recommended.

FA is an autosomal recessive neurodegenerative disorder. Given the progressive nature of the disorder and its end-organ manifestations, various surgical procedures may be required in these patients. The main points of perioperative management for FA patients are the preoperative identification and evaluation of associated co-morbid conditions which may impact the perioperative care. The potential comorbid conditions include HCM, diabetes mellitus, and restrictive lung disease.

\section{References}

1. Friedman LS, Farmer JM, Perlman S, Wilmot G, Gomez $\mathrm{CM}$, Bushara KO, Mathews KD, et al. Measuring the rate of progression in Friedreich ataxia: implications for clinical trial design. Mov Disord. 2010;25(4):426-432.

2. Koeppen AH. Friedreich's ataxia: pathology, pathogenesis, and molecular genetics. J Neurol Sci. 2011;303(12):1-12.

3. Delatycki MB, Williamson R, Forrest SM. Friedreich ataxia: an overview. J Med Genet. 2000;37(1):1-8.

4. Leonard H, Forsyth R. Friedreich's ataxia presenting after cardiac transplantation. Arch Dis Child. 2001;84(2):167-168.

5. Milbrandt TA, Kunes JR, Karol LA. Friedreich's ataxia and scoliosis: the experience at two institutions. J Pediatr Orthop. 2008;28(2):234-238.

6. Mottram PM, Delatycki MB, Donelan L, Gelman JS, Corben L, Peverill RE. Early changes in left ventricular long-axis function in Friedreich ataxia: relation with the FXN gene mutation and cardiac structural change. J Am Soc Echocardiogr. 2011;24(7):782-789.

7. Rajagopalan B, Francis JM, Cooke F, Korlipara LV, Blamire AM, Schapira AH, Madan J, et al. Analysis of the factors influencing the cardiac phenotype in Friedreich's ataxia. Mov Disord. 2010;25(7):846-852.

8. Haering JM, Comunale ME, Parker RA, Lowenstein E, Douglas PS, Krumholz HM, Manning WJ. Cardiac risk of noncardiac surgery in patients with asymmetric septal hypertrophy. Anesthesiology. 1996;85(2):254-259.

9. Tobias JD. Etomidate: applications in pediatric critical care and pediatric anesthesiology. Pediatr Crit Care Med. 2000;1(2):100-106.

10. Soliman DE, Maslow AD, Bokesch PM, Strafford M, Karlin L, Rhodes J, Marx GR. Transoesophageal echocardiography during scoliosis repair: comparison with CVP monitoring. Can J Anaesth. 1998;45(10):925-932.

11. Vincent JL. Does central venous oxygen saturation accurately reflect mixed venous oxygen saturation? Nothing is simple, unfortunately. Intensive Care Med. 1992;18(7):386-387.

12. Bernard JM, Le Penven-Henninger C, Passuti N. Sudden decreases in mixed venous oxygen saturation during posterior spinal fusion. Anesth Analg. 1995;80(5):10381041.

13. Tobias JD. Cerebral oxygenation monitoring: near-infrared spectroscopy. Expert Rev Med Devices. 2006;3:235243.

14. Martyn JA, Richtsfeld M. Succinylcholine-induced hyperkalemia in acquired pathologic states: etiologic factors and molecular mechanisms. Anesthesiology. 2006;104(1):158-169.

15. Pancaro C, Renz D. Anesthetic management in Friedreich's ataxia. Paediatr Anaesth. 2005;15(5):433-434.

16. Ozgul U, Erdogan MA, Aydogan MS, Korkmaz MF, Nakir H, Durmus M. Anesthesia management and Friedreich's Ataxia. Med Science. 2013;2:928-934.

17. Tobias JD. Strategies for minimizing blood loss in orthopedic surgery. Semin Hematol. 2004;41(1 Suppl 1):145- 
156.

18. Almenrader N, Patel D. Spinal fusion surgery in children with non-idiopathic scoliosis: is there a need for routine postoperative ventilation? $\mathrm{Br} \mathrm{J}$ Anaesth.
2006;97(6):851-857.

19. Pelosi P, Jaber S. Non-invasive respiratory support in the perioperative period. Curr Opin Anaesthesiol. 2010;3:2233-2228. 